\title{
Exploring the Morphology of Anterior Interosseous Nerve and Relating It to Its Clinical Conditions
}

\author{
Yelluru Lakshmisha RAO ${ }^{1}$, Mangala M. PAI ${ }^{1}$, Peruvaje R. KRISHNAPRASAD ${ }^{2}$, \\ Bukkambudhi V. MURLIMANJU' ${ }^{1}$, Tonse MAMATHA ${ }^{1}$, Latha V. PRABHU ${ }^{1}$ \\ ${ }^{1}$ Kasturba Medical College, Mangalore, Manipal Academy of Higher Education, Department of Anatomy, Manipal, Karnataka, India \\ ${ }^{2}$ Manipal Academy of Higher Education, Kasturba Medical College and Kasturba Hospital, Department of Orthopaedics, Manipal, Karnataka, \\ India \\ This study has been presented at the $67^{\text {th }}$ National Conference of the Anatomical Society of India between 17 and 20 November 2019 at New Delhi, \\ India. Theme: "Anatomy Sans Frontiers", which was held in Vardhman Mahavir Medical College, Safdarjung Hospital.
}

Corresponding author: B.V. MURLIMANJU flutemist@gmail.com

\section{ABSTRACT}

AIM: To study topography and variability in the origin of anterior interosseous nerve; to identify the branching pattern of the anterior interosseous nerve supplying the flexor digitorum profundus, flexor pollicis longus, and pronator quadratus muscles.

MATERIAL and METHODS: The present study included 70 formalin-fixed upper limbs of adult human cadavers. The origin of the anterior interosseous nerve was categorized into 3 types. The morphometric data obtained in this study were represented as mean \pm $\mathrm{SD}$ and the dimensions were given in millimeter. The measurements were compared statistically by using 'EZR software, version $1.38,2019$ '. The 'paired t-test' was applied and the ' $p$ ' value less than 0.05 was considered as statistically significant.

RESULTS: It was observed that the origin of the anterior interosseous nerve was extremely variable. It was ranging from the midepicondylar point of the elbow joint up to as below as $86 \mathrm{~mm}$ from it. The distance of its origin from the midpoint of the pronator teres muscle ranged between $70 \mathrm{~mm}$ above the pronator teres muscle to $22 \mathrm{~mm}$ below it. In one of the forearms, the median nerve supplied the medial two tendons of the FDP, instead of the ulnar nerve.

CONCLUSION: The present study provided additional information about the origin, topography, and distribution of the anterior interosseous nerve. The data will provide further insight into the causes of nerve compression syndromes. It will also help in planning the surgical approach into the distal humerus, elbow joint, and proximal ends of radius and ulna, without causing any nerve injury.

KEYWORDS: Compartment syndromes, Median nerve disease, Median nerve, Anterior Interosseous Nerve, Neuropathy

ABBREVIATIONS: AIN: Anterior interosseous nerve, BA: Brachial artery, BR: Brachioradialis muscle, FDP: Flexor digitorum profundus, FDS: Flexor digitorum superficialis, FPL: Flexor pollicis longus, MN: Median nerve, PQ: Pronator quadratus, PT: Pronator teres, UN: Ulnar nerve

\section{INTRODUCTION}

A nterior interosseous nerve (AIN) is a motor branch of the median nerve (MN), which supplies the deep flexor muscles of the forearm (19). The MN below the distal border of the pronator teres (PT) muscle, where it leaves the cubital fossa provides the AIN $(14,19)$. However, it was reported that the origin of AIN varies and the relation between AIN and PT muscle is also often variable (2). Such variant origin and the course of AIN are noteworthy during
Yelluru Lakshmisha RAO

Mangala M. PAI

(D) : 0000-0002-9951-1606

Peruvaje R. KRISHNAPRASAD (i) : 0000-0002-3595-6227
Bukkambudhi V. MURLIMANJU (10): 0000-0003-1248-8296

Tonse MAMATHA

Latha V. PRABHU
(D) : $0000-0003-1322-6730$

(1) : 0000-0002-1386-0099 
the diagnosis and treatment of AIN neuropathies. In $23 \%$ of people, communication between AIN and $\mathrm{MN}$ or ulnar nerve (UN) can be seen, which is known as Martin-Gruber anastomosis (16). AIN is commonly involved as a part of brachial plexus injury, supracondylar fracture of humerus, and Parsonage-Turner syndrome $(14,17)$. It was described that AIN palsy is comparatively uncommon and can manifest as partial or complete paralysis of deep flexor muscles (17). The common causes of AIN injury include trauma, iatrogenic during surgery, sling, and dressing (15).

The AIN supplies flexor pollicis longus (FPL), pronator quadratus (PQ), and flexor digitorum profundus (FDP) of middle and index fingers. Whereas half of the FDP, which gives tendons to the ring and little fingers is supplied by the UN. It was reported that the distribution of AIN and UN are variable (2) and the knowledge of this variation is clinically important. Since symptoms of AIN palsy mimic the ligamentous injury of the finger, the diagnosis of AIN injury or injury to its branches is clinically a challenging task (1). It is described that identifiable superficial landmarks of AIN will aid the surgeons in isolating the nerve and avoiding complications during or after the surgeries like a reduction of forearm fractures and nerve release procedures (21). It was observed that there are very few anatomical reports about the course and branching pattern of AIN in the scientific literature. Since AIN is located deep in the forearm, injury to this nerve is not common, however, its injury can lead to many complications and disabilities to the patient. The knowledge of variable origin, course and distribution of AIN will be enlightening to the clinical practice. In this context, the present study aimed to study topography and variability in the origin of AIN, to measure the distance of its origin from the mid epicondylar point and the midpoint of PT muscle. The objective was also to study the branching pattern of AIN in supplying the FDP, FPL, and PQ muscles.

\section{MATERIAL and METHODS}

The present study was performed on 70 formalin-fixed forearms of adult human cadavers. Among them, 37 were right-sided and 33 were left-sided specimens. The forearms with external visible pathological changes were excluded. The extremities were meticulously dissected and the pattern of origin and muscular branches of AIN were carefully observed and noted. The origin of AIN was categorized into 3 types (Figure 1) as below:

Type 1 - the origin of AIN above the level of the midpoint of PT muscle,

Type 2 - the origin of AIN deep to the PT muscle,

Type 3 - the origin of AIN below the level of the midpoint of PT muscle.

The ethics committee of our institution has approved this descriptive cross-sectional study (Date: 21.08.2019; IEC KMC MLR 08-19/354). The morphometric data obtained in this study were represented as mean \pm SD and the dimensions were given in millimeter. The right and left-sided measurements were compared statistically by using the 'EZR software, version 1.38, 2019' (12). The data were statistically compared by using the 'paired t-test' and the ' $p$ ' value less than 0.05 was considered as statistically significant.

\section{RESULTS}

The present study observed that the origin of AIN was extremely variable. Its origin was ranging from the midepicondylar point of the elbow joint up to as below as $86 \mathrm{~mm}$ from it. The distance of its origin from the midpoint of PT muscle ranged between $70 \mathrm{~mm}$ above and $22 \mathrm{~mm}$ below it. The mean length of the forearm of the cadaveric specimens, the distance of the origin of AIN from midepicondylar point, and the distance of the first branch of AIN to FPL, FDP, and PQ from its origin was tabulated separately for the right and left-sided specimens (Table I). The analysis showed that there was no statistical significance between the right and left sides ( $p>0.05$ ).

The distance of the first branch of AIN to FPL and FDP was also analyzed (Table II) and this was also statistically not significant ( $p>0.05$ ). It was observed that, in $36.3 \%$ cases, branches to FPL were topographically located at $0-30 \mathrm{~mm}$ away, in $47.6 \%$ cases, $31-60 \mathrm{~mm}$ away, and in $11.9 \%$ cases, the branches were arising between 61-90 mm away from the origin of AIN. They were located $91-110 \mathrm{~mm}$ away from the AIN origin in the remaining $4.2 \%$ cases.

The range of distance of branches of AIN to FDP was also analyzed. It was observed that $54.6 \%$ of branches were topographically located at $0-30 \mathrm{~mm}$ away from the origin of AIN and $31-60 \mathrm{~mm}$ away in $27.6 \%$ cases. The branches were observed between the distance of $61 \mathrm{~mm}$ and $90 \mathrm{~mm}$ in $15.4 \%$ cases. In the remaining $2.4 \%$ cases, the branches to FDP were arising 91-120 $\mathrm{mm}$ away from the origin of AIN.

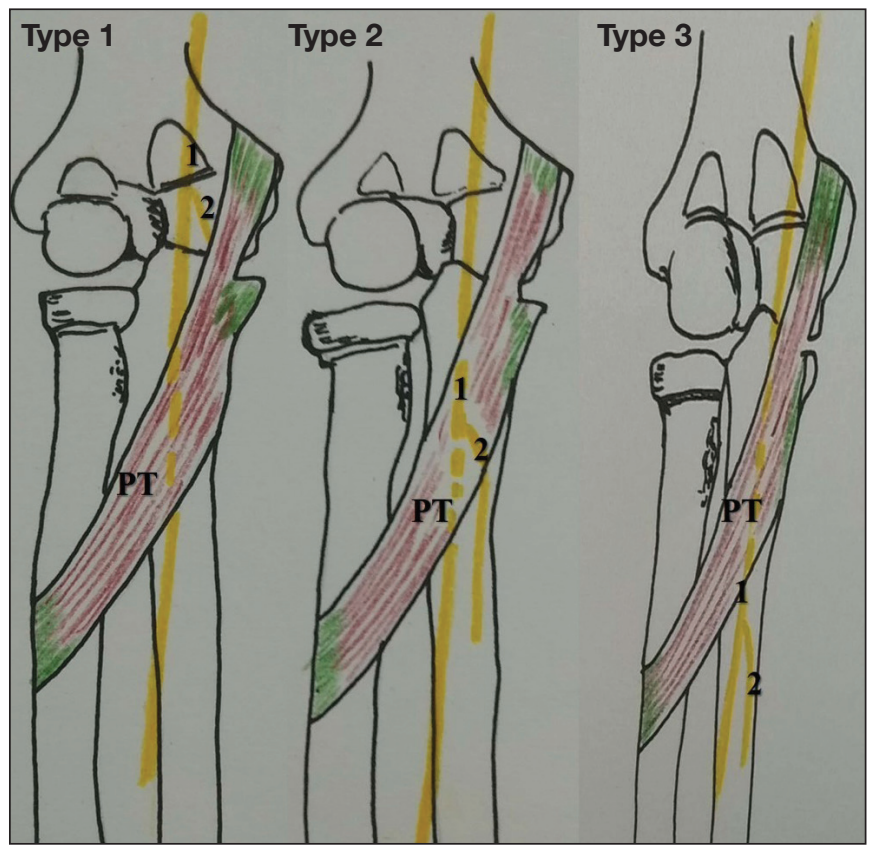

Figure 1: Variability in the origin of the anterior interosseous nerve (AIN) (PT-pronator teres muscle, 1-median nerve, 2-AIN). 
Table I: Comparison of the Data of the Present Study Over the Right And Left Sides $(n=70)$

\begin{tabular}{lccc}
\hline Parameter Measured & Left Side $(\mathbf{n = 3 7 )}$ & Right Side $(\mathbf{n}=\mathbf{3 3})$ & $\mathbf{p}$ \\
\hline Length of forearm & $240.82 \pm 45.41$ & $259.85 \pm 25.73$ & $33.09 \pm 20.71$ \\
\hline Distance of origin of AIN from midepicondylar point & $43.5 \pm 21.03$ & $35.85 \pm 19.9$ \\
\hline Distance of first branch of AIN to FPL from its origin & $29.78 \pm 16.78$ & $37.09 \pm 23.9$ \\
\hline Distance of first branch of AIN to FDP from its origin & $30.10 \pm 23.15$ & $143.61 \pm 27.8$ & 0.32 \\
\hline Distance of first branch of AIN to PQ from its origin & $128.73 \pm 27.0$ & 0.06 \\
\hline
\end{tabular}

Measurements are in $\mathrm{mm}$, values expressed as mean $\pm S D$, Statistical analysis - paired ' $t$ ' test.

Table II: Comparison of Distance of the First Branch of AIN to FPL and FDP $(\mathrm{n}=70)$

\begin{tabular}{ccc}
\hline FPL & FDP & p \\
\hline $32.97 \pm 18.50$ & $33.77 \pm 23.54$ & 0.77 \\
\hline
\end{tabular}

Measurements are in $\mathrm{mm}$, values expressed as mean $\pm S D$, Statistical analysis - paired ' $t$ ' test.

The frequency of various types of origin of AIN from the MN was determined. In $48.6 \%$ cases, type 1 pattern (Figure $2 \mathrm{~A}$ ), $31.4 \%$ cases, type 2 (Figure $2 \mathrm{~B}$ ) and in $20 \%$ cases (Figure $2 \mathrm{C}$ ), type 3 pattern was observed. It was observed that $40 \%$ of the FPL had two branches, $30 \%$ had a solitary branch, $26 \%$ had triple branches and $4 \%$ had four branches arising from the AIN. The AIN gave three branches to FDP in $45.7 \%$ cases, two branches in $24.3 \%$, solitary branch in $24.3 \%$, and four branches in $5.7 \%$ cases. It was observed that $91.4 \%$ of the PQ had a solitary branch supplying it, and only $8.6 \%$ cases had double branches from the AIN. The branches of AIN to FDP and FPL are represented in Figure 3. In one of the forearms, the medial two tendons of the FDP were supplied by the MN instead of the UN.

\section{DISCUSSION}

The AIN is essentially a motor nerve and it is the only motor innervation to the deep muscles of the forearm (22). However, the medial tendons of FDP are supplied by the UN. Its origin is about $5-8 \mathrm{~cm}$ distal to the lateral epicondyle of humerus, but its fibers can be traced back to the brachial plexus (20). AIN courses by piercing or running beneath the PT muscle and appears anterior to the interosseous membrane until the wrist joint. AIN provides motor supply to the PQ, FDP to the index and middle fingers and FPL (22). The AIN palsy is a rare condition with unknown etiology and pathophysiology (3). It was Duchenne de Boulogne, who gave the first description of AIN palsy (5). There are lots of anatomical reasons for the AIN compression, which include a tendinous origin of the deep head of PT and flexor digitorum superficialis (FDS) muscles, accessory tendons from the FDS, accessory head of FPL and tendinous origin of flexor carpi radialis brevis (18). AIN compression due to the bone and soft tissue are included under the non-traumatic nerve injury (13). The anatomical knowledge of AIN is important because it can be injured iatrogenically due to surgical dressings, slings, venepuncture, and surgical operations (22).

The patients with AIN compression can present with weakness in FDP tendons to the index and middle fingers, $P Q$ muscle, and flexion of the distal phalanx of the thumb. This can be diagnosed by procedures like electromyogram and nerve conduction studies (13). The patients with AIN palsy will have the inability to make the 'OK' sign. This can be demonstrated by asking the patient to flex his interphalangeal joint of the thumb and distal interphalangeal joint of the index finger. AIN can also be tested by asking the patient to pinch a sheet of paper between the thumb and index finger by using the fingertips only. The paper will be tried pulling and if the patient cannot hold the paper with fingertips, it suggests the AIN injury. Sometimes this is compensated by using an adaptive grip, in which the interphalangeal joint of the thumb and distal interphalangeal joint of the index finger will remain extended (4). Injury to the AIN can be complete or incomplete. In the case of complete AIN palsy, all the deep flexor muscles of the forearm which are supplied by it are affected, whereas, in case of incomplete palsy, only FPL or FDP of the index finger are affected. The clinical manifestations of AIN palsy depend upon the location and degree of axonal damage (16). AIN palsy is also known as Kiloh- Nevin syndrome, which includes pain in the proximal forearm associated with a palsy of all or any muscle, which is innervated by the AIN. The common cause of Kiloh-Nevin syndrome is the nerve entrapment. Though the origin of AIN is below the elbow joint, the supracondylar fractures can also result in the AIN palsy, since the fibers of AIN can be isolated at the level of brachial plexus (10).

Vincelet et al. opined that post supracondylar AIN palsy is due to the injury to $\mathrm{MN}$ which contains the fascicles of AIN (23). According to them, $6.6-31 \%$ of supracondylar fractures will have neuropathies and one-third of these are related to AIN. The surgical treatment of supracondylar fracture of the humerus is usually performed through the posterior approach. However, if this fracture has an associated brachial artery injury, then the cubital approach is practiced. Identification and preservation of the neurovascular structures including the AIN are important during the dissection of the cubital fossa. The AIN can also be injured during the surgical repair of the fractured shaft of radius and Monteggias fracture. Hence preserving the AIN with its muscular branches is crucial to the orthopedic surgeons, while performing these surgeries. 

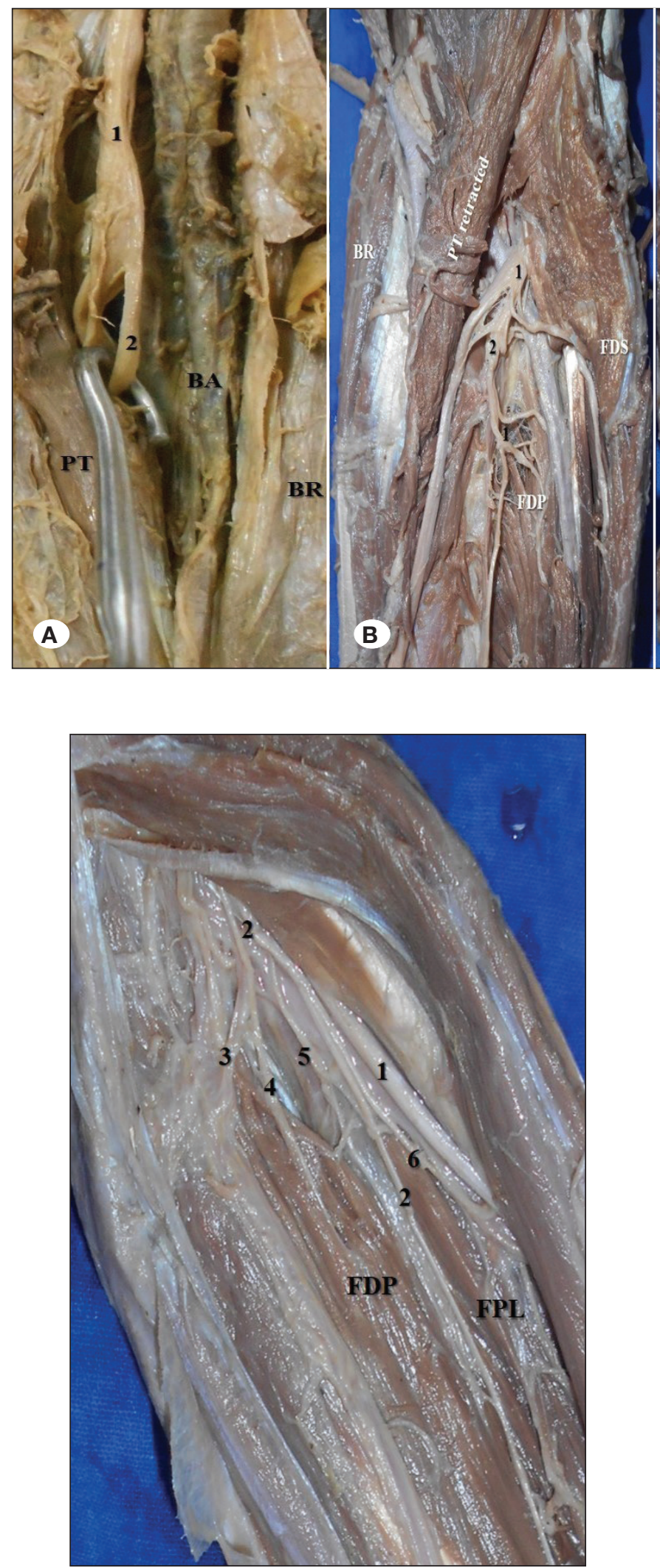

Figure 3: Cadaveric forearm showing the branches of AIN to the FDP and FPL (FDP-flexor digitorum profundus muscle; FPL-flexor pollicis longus muscle, 1-median nerve; 2-anterior interosseous nerve, 3,4,5- branches to FDP, 6-branch to FPL).

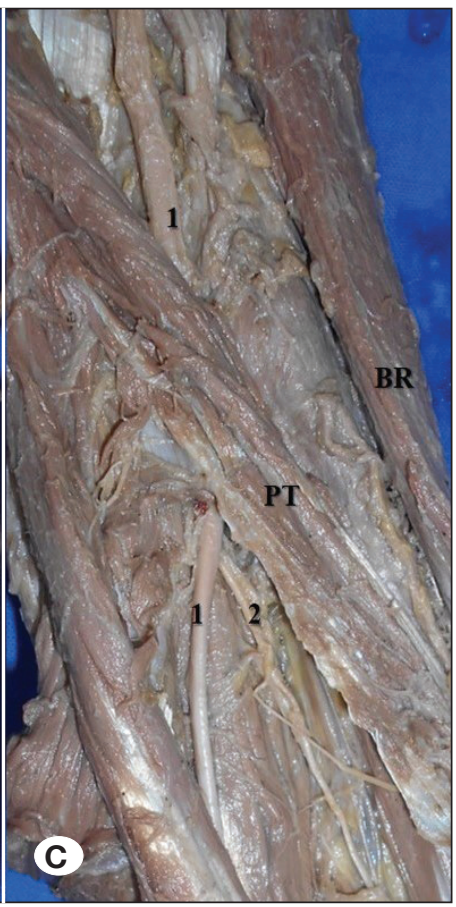

Figure 2: Origin of AIN from the median nerve; $\mathbf{A}$ ) above the level of the midpoint of pronator teres, type 1 pattern (48.6\% cases); $\mathbf{B})$ deep to the pronator teres, type 2 pattern (31.4\% cases); C) below the level of the midpoint of pronator teres, type 3 pattern, observed in $20 \%$ cases (1-median nerve; 2-anterior interosseous nerve;

BA: brachial artery;

BR: brachioradialis muscle;

PT: pronator teres muscle;

FDS: flexor digitorum

superficialis muscle; FDP: flexor digitorum profundus muscle).

El Domiaty et al. observed that the FPL and FDP are innervated mainly by the AIN and rarely by the MN (6). The classical distribution of nerve supply to FDP is that its radial two slips, to index and middle fingers are supplied by $\operatorname{AIN}(\mathrm{C} 8, \mathrm{~T} 1)$, and ulnar slips to ring and little fingers are supplied by the UN (C7). But the innervation of FDP is highly variable and some slips of FDP may get dual nerve supply (9). In the present study, there was one case in which the tendons of FDP to ring and little fingers were supplied by the MN instead of the UN. The AIN supplies $P Q$ before entering the muscle or when it passes through the muscle. AIN also gives branches to the distal radio-ulnar joint, usually before giving muscular branches to $P Q$ (8). The AIN is important to plastic surgeons as this can be used as nerve transfer to the thenar branch of MN. Here the nerve can be harvested at the proximal border of $P Q$ muscle (7). Jarvie et al. used the AIN transfer to the UN in case of ulnar neuropathy (11). During the harvesting of AIN, its course and distribution should be studied carefully.

The literature survey did not reveal much of anatomical studies of AIN, though its clinical interest is wider. The data about the morphology of AIN are not available from the Indian literature. In this present study, AIN was examined from anatomical samples and it was observed that it had variable origin from MN. This study described the frequency of the topographical distribution of the origin of AIN. The distance of its origin from the midpoint of the PT muscle and the midepicondylar point were measured. There were no anatomical studies, which checked the distribution of AIN to the deep muscles of the forearm. This present study calculated the distance of the first branch of AIN to the FPL, FDP, and PQ muscles from its origin from MN. It was observed that type 1 pattern of origin was the most common variety $(48.6 \%)$, in which the AIN originated above the level of the midpoint of PT. There were multiple 
branches of AIN which were supplying the deep muscles of the forearm. It was common to see FPL having double branches (40\%) and FDP with triple branches (45.7\%). It was observed that PQ had a solitary branch of AIN in $91.4 \%$ cases. It was not possible to do a comparison of the data of this study with the previous studies of the same subject, because they are not available in the literature.

\section{CONCLUSION}

The present study had provided additional information about the variability in origin and the topographical distribution of the branches of AIN to the deep muscles of the forearm. These data can be considered as a novel in the anatomical literature. The findings of this study will provide knowledge about the etiopathogenesis of nerve entrapment syndromes of the forearm. The data will assist the surgeons in planning the anterior approach to the forearm and elbow joint, without causing the iatrogenic injury to the AIN.

\section{REFERENCES}

1. Akhondi $\mathrm{H}$, Varacallo $\mathrm{M}$ : Anterior Interosseous Syndrome. In: StatPearls [Internet]. Treasure Island (Florida): StatPearls Publishing, 2020

2. Caetano EB, Vieira LA, Sabongi Neto JJ, Caetano MBF, Sabongi RG: Anterior interosseous nerve: Anatomical study and clinical implications. Rev Bras Ortop 53:575-581, 2018

3. Chi Y, Harness NG: Anterior interosseous nerve syndrome. J Hand Surg 35:2078-2080, 2010

4. Dang AC, Rodner CM: Unusual compression neuropathies of the forearm, part II: Median nerve. J Hand Surg 34:1915-1920, 2009

5. Duchenne de Boulogne GBA: De l'e'lectrisation localise'e. vol 1. 3rd Ed. J.-B. Baillie're, Paris, 1872

6. El Domiaty MA, Zoair MM, Sheta AA: The prevalence of accessory heads of the flexor pollicis longus and the flexor digitorum profundus muscles in Egyptians and their relations to median and anterior interosseous nerves. Folia Morphol (Warsz) 67:63-71, 2008

7. Frank K, Englbrecht M, Koban KC, Cotofana SC, Stewart JK, Giunta RE, Schenck TL: Nerve transfer of the anterior interosseous nerve to the thenar branch of the median nerve - an anatomical and histological analysis. J Plast Reconstr Aesthet Surg 72:751-758, 2019

8. Hohenberger GM, Maier MJ, Dolcet C, Weiglein AH, Schwarz A, Matzi V: Sensory nerve supply of the distal radio-ulnar joint with regard to wrist denervation. J Hand Surg Eur 42:586591,2017
9. Hwang K, Bang SJ, Chung SH: Innervation of the flexor digitorum profundus: A systematic review. Plast Surg (Oakv) 26:120-125, 2018

10. Jain M, Nayak C, Samal BP, Tirpude A: Kiloh-Nevin syndrome associated with humeral shaft fracture. BMJ Case Rep 2018: bcr-2018-226506, 2018

11. Jarvie G, Hupin-Debeurme M, Glaris Z, Daneshvar $P$ : Supercharge end-to-side anterior interosseous nerve to ulnar motor nerve transfer for severe ulnar neuropathy: Two cases suggesting recovery secondary to nerve transfer. J Orthop Case Rep 8:25-28, 2018

12. Kanda Y: Statistical analysis using freely-available "EZR (Easy R)"software. Rinsho Ketsueki 56:2258-2266, 2015

13. Kim DH, Murovic JA, Kim YY, Kline DG: Surgical treatment and outcomes in 15 patients with anterior interosseous nerve entrapments and injuries. J Neurosurg 104:757-765, 2006

14. Mauricio EA, Rubin DI: Inclusion body myositis mimicking bilateral anterior interosseous neuropathies. J Hand Surg Am 44:1100.e1-1100.e4, 2019

15. Nammour M, Desai B, Warren M, Sisco-Wise L: Anterior interosseous nerve palsy after shoulder arthroscopy treated with surgical decompression: A case series and systematic review of the literature. Hand (NY) 2019 (Online ahead of print)

16. Raoulis V, Tsoucalas G, Batsiou A, Zibis AH: An uncommon case of atraumatic palsy of a branch of the anterior interosseous nerve with a late spontaneous recovery. Int $\mathrm{J}$ Appl Basic Med Res 9:182-184, 2019

17. Shinohara T, Takahashi S, Hirata H: Anterior interosseous nerve palsy mimicking rupture of the index flexor digitorum profundus after volar locking plate fixation of a distal radius fracture. Nagoya J Med Sci 79:421-425, 2017

18. Spinner M: Injuries to the major branches of peripheral nerves of the forearm. Philadelphia: WB Saunders, 1978:160-227

19. Standring S: Gray's Anatomy: The anatomical basis of clinical practice, $40^{\text {th }}$ ed, New York: Elsevier Churchill Livingstone, 2005: 826

20. Sunderland S: The innervation of the flexor digitorum profundus and lumbrical muscles. Anat Rec 93:317-321, 1945

21. Tubbs RS, Custis JW, Salter EG, Wellons JC 3rd, Blount JP, Oakes WJ: Quantitation of and superficial surgical landmarks for the anterior interosseous nerve. J Neurosurg 104:787-791, 2006

22. Ulrich D, Piatkowski A, Pallua N: Anterior interosseous nerve syndrome: Retrospective analysis of 14 patients. Arch Orthop Trauma Surg 131:1561-1565, 2011

23. Vincelet $Y$, Journeau P, Popkov D, Haumont T, Lascombes $P$ : The anatomical basis for anterior interosseous nerve palsy secondary to supracondylar humerus fractures in children. Orthop Traumatol Surg Res 99:543-547, 2013 